\title{
ALL-SOLID-STATE PICOSECOND LASER SYSTEM FOR PHOTO CATHODE RF-GUN AND X-RAY GENERATION AT WASEDA UNIVERSITY
}

\author{
T. Oshima, Y. Hama, H. Ishikawa, S. Kashiwagi, R. Kuroda, M. Washio, A. Yada, \\ Waseda University, Tokyo, Japan \\ H. Hayano, J. Urakawa, KEK, Ibaraki, Japan
}

\begin{abstract}
An 1.6 cell rf-gun system has been installed at Waseda University for the generation of high charge, short pulse and low emittance electron beam. The gun is one of essential component for X-ray generation using inverse Compton scattering between photo electron bunch and laser pulse. An all-solid-state picosecond Nd:YLF laser system has been installed at Waseda University and will be used not only for photo electron generation in the rf-gun but also for collision with the photo electron bunch. The laser system has the frequency conversion section, which is operated to produce the UV (262 nm) using BBO crystals from the amplified fundamental $(1047 \mathrm{~nm})$. Extremely stable laser system is required for the generation of high quality electron beam, and the system is essential component for the generation of X-rays through the inverse Compton scattering. Timing and intensity stabilities of laser pulse have been measured using time domain demodulation technique. As the results, timing stability of $0.26 \mathrm{ps}$ against to the local oscillator of $\mathrm{rf}$ and intensity stability of $0.11 \%$ have been achieved. We have carried out the numerical simulation to calculate the number of photons and the energies of X-rays by changing the crossing angles.
\end{abstract}

\section{INTRODUCTION}

Recently, photo cathode rf-gun is developing at many institutes for the generation of high quality electron beam. The rf-gun has many advantages: (I) Beam time structure can be controlled by laser system. (ii) Buncher system is not necessary. (iii) Low emittance beam can be produced applying high accelerating field in rf cavities. High quality electron beam is useful for many applications.

An 1.6 cell rf-gun system has been installed at Waseda University for the generation of high charge, short pulse and low emittance electron beam. Our system consists of high quality laser, an rf source and an rf cavity with $\mathrm{Mg}$ cathode. The rf power source is $10 \mathrm{MW}$ S-band klystron (Tomson:TV2019B6) and a small pulse modulator (Nissin Electric Co., Ltd.) which has good stability and flatness of the output pulse. It is possible to produce high intensity electron beam because of high quantum efficiency of $\mathrm{Mg}$ cathode. The rf cavity is manufactured using diamond turning method for decreasing in dark current due to field emission under the high gradient operation. We will start the short pulsed X-ray generation experiment using inverse
Compton scattering between laser pulse and photo electron bunch for one of applications of our system.

Characteristics of photo electron beam is strongly depended by laser pulse stability, e.g., timing stability, intensity stability and pointing stability. Especially, timing and intensity stabilities of laser pulse are studied using timing domain demodulation technique. In order to avoid the instability of irradiation point on the photo cathode, we have set the laser system close to the rf-gun cavity. Therefore, we may suffer somewhat serious noise problems for the timing and intensity stabilities due to rf and radiation noises. Hence, we have investigated the effects of these noises on above two stabilities. The results of simulation study on the generation of X-rays through the inverse Compton scattering is also introduced.

\section{LASER SYSTEM}

\section{$2.1 \quad \mathrm{Nd}:$ YLF laser and synchronisation system}

An all-solid-state Nd:YLF laser system has been installed at Waseda University and will be used not only for photo electron generation in the rf-gun but also for collision with the photo electron bunch. We have chosen PULRISE V Nd:YLF laser (Sumitomo Heavy Industries, Ltd.) as the laser system $[1,2]$ (pulse width is $10 \mathrm{ps).} \mathrm{This} \mathrm{laser} \mathrm{system}$ consists of passively mode-locked oscillator part (including timing stabiliser system), regenerative amplifier part and frequency conversion part (with amplitude stabiliser system). Figure 1 shows PULRISE V laser system.

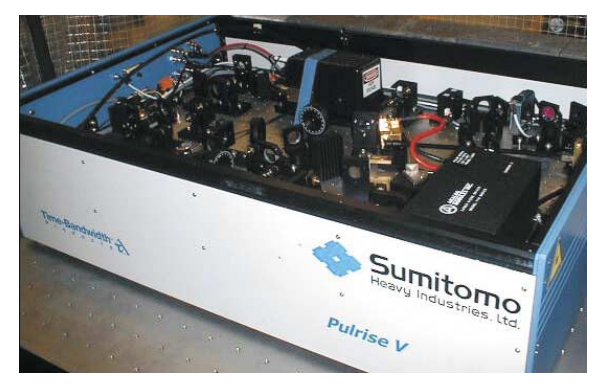

Figure 1: Photograph of PULRISE V

Seed laser is diode-pumped Nd:YLF laser. It is amplified by regenerative amplifier with Pockels Cell. Doublet BBO $\left(\mathrm{BaB}_{2} \mathrm{O}_{4}\right)$ crystals convert the amplified IR as a fundamental (wavelength of $1047 \mathrm{~nm}$ and intensity of 4 
$\mathrm{mJ} /$ pulse) to the green as a second harmonics (wavelength of $524 \mathrm{~nm}$ and intensity of $2.5 \mathrm{~mJ} /$ pulse) and the UV as a fourth harmonics (wavelength of $262 \mathrm{~nm}$ and intensity of $0.6 \mathrm{~mJ} /$ pulse).

The laser-driven photo cathode rf-gun requires precise timing synchronisation between laser injection and rf signal for rf-gun cavities. The inverse Compton scattering experiment needs good timing precision between photo electron bunch and laser pulse. The UV is fourth harmonics of the IR. Reference rf signals of laser system $(119 \mathrm{MHz}$ and $25 \mathrm{~Hz}$ ) are synchronised rf of $2856 \mathrm{MHz}$ (shown in figure 2). The UV, photo electron beam and the IR are precisely synchronised rf signal of rf-gun cavities down to 1 ps.

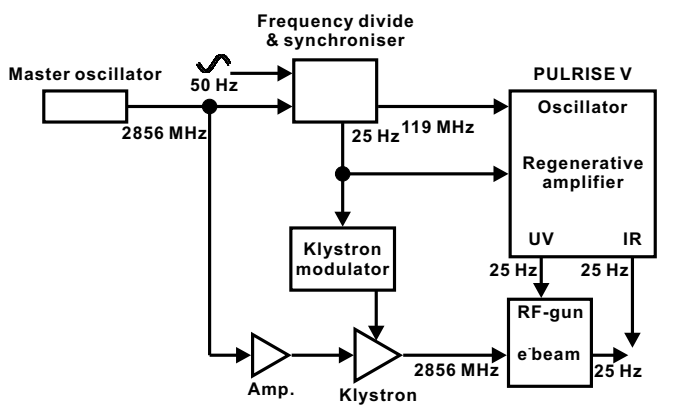

Figure 2: Synchronisation system

\subsection{Stabiliser system}

PULRISE V has two different stabilisers using feedback systems. One is a timing stabiliser. The other is an intensity stabiliser. The timing stabiliser, which is active compensation system in oscillator part, tunes the seed laser phase against temperature change and the timing jitter from reference rf signal $(119 \mathrm{MHz})$. The intensity stabiliser for the UV is located in frequency conversion part, and modulates output power of the UV. There are no stabilisers for the IR and the green.

\section{MEASUREMENTS}

\subsection{Time domain demodulation technique}

The measurements of timing and intensity stabilities of seed laser have been demonstrated by vector signal analyser (VSA). VSA can measure timing and amplitude jitters by time domain demodulation technique (TDDT) [3]. Pulse intensity is given by,

$$
V(t)=\sum_{n=1}^{\infty} v_{n}[1+\varepsilon(t)] \sin \left[n\left\{2 \pi f_{r} t+\phi(t)\right\}\right],
$$

where $v_{n}$ is intensity, $f_{r}$ is repetition rate, $\varepsilon(t)$ and $\phi(t)$ are amplitude and phase noise, and $\mathrm{n}$ is order of harmonics. Power spectral densities (PSD) of $\varepsilon(t)$ and $\phi(t)$ are obtained by TDDT. PSD is obtained by Fourier conjugation of autocorrelation of a function. The rms timing and amplitude jitters, as a function of PSD, are expressed as follows.

$$
\begin{aligned}
\sigma_{T J} & =\frac{1}{2 \pi f_{r}} \sqrt{\int_{f_{l}}^{f_{h}} S_{\phi}(f) \mathrm{d} f} \\
\sigma_{A J} & =\sqrt{\int_{f_{l}}^{f_{h}} S_{\varepsilon}(f) \mathrm{d} f}
\end{aligned}
$$

where $S_{\phi}(f)$ and $S_{\varepsilon}(f)$ are PSD of $\phi(t)$ and $\varepsilon(t)$ and $f_{h}$ and $f_{l}$ are higher and lower limits of frequency range.

\subsection{Results and Discussion}

In order to investigate the effect of $\mathrm{rf}$ and radiation on the timing and intensity stabilities, we have measured timing and amplitude jitters at two conditions. One is RF-OFF condition, which means measurement with no rf field in $\mathrm{rf}$ cavity. The other is RF-ON condition, which means supplying rf power to rf-gun.
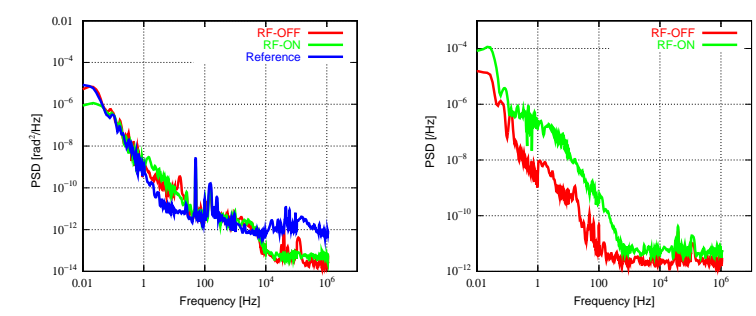

Figure 3: Left : timing jitter. Right : amplitude jitter. PSD of $\phi$ and $\varepsilon$ as functions of frequency

Figure 3 shows PSD of $\phi(t)$ and $\varepsilon(t)$ as functions of frequency. Integration of PSD gives timing and amplitude jitter (equation (2),(3)). Timing jitter was obtained as 0.25 ps with no rf power and 0.26 ps with rf power in cavity. Hence, it is clear that we can avoid the timing jitter problem of the laser. On the other hand, amplitude jitter was obtained as $0.03 \%$ without rf power and $0.11 \%$ with rf power in cavity. This means that $\mathrm{rf}$ and radiation noises should affect on the laser light intensity. Therefore, noise shielding will be installed before routine operations.

\section{X-RAY GENERATION}

Development of X-ray generation system using inverse Compton scattering $[4,5]$ between stable laser pulse and electron bunch will start at Waseda University in 2001. Parameters of electron bunch and laser pulse are shown in Table 1.

Energy of X-rays is given by following equation.

$$
E_{X}=\frac{E_{l} E_{e}(1-\beta \cos (\varphi))}{E_{l}(1+\cos (\varphi-\theta))+E_{e}(1-\beta \cos (\theta))}
$$

where $E_{l}$ and $E_{e}$ are energies of laser photon and electron, $\varphi$ is crossing angle between laser pulse and electron bunch and $\theta$ is scattered angle of X-rays. 
Table 1: Parameters of electron bunch and laser pulse

\begin{tabular}{|c|c|}
\hline \multicolumn{2}{|l|}{ Electron bunch } \\
\hline Beam energy & $5.0[\mathrm{MeV}]$ \\
\hline Bunch charge & 3 [nC/bunch] \\
\hline Bunch length (FWHM) & $10[\mathrm{ps}]$ \\
\hline Beam size at focal point $(\sigma \mathrm{x} / \sigma \mathrm{y})$ & $100 / 100[\mu \mathrm{m}]$ \\
\hline \multicolumn{2}{|l|}{ Laser pulse } \\
\hline Wavelength & $1047[\mathrm{~nm}]$ \\
\hline Intensity & 4 [mJ/pulse] \\
\hline Pulse length (FWHM) & 10 [ps] \\
\hline Beam size at focal point $(\sigma \mathrm{x} / \sigma \mathrm{y})$ & $30 / 30[\mu \mathrm{m}]$ \\
\hline
\end{tabular}

The inverse Compton scattering with different crossing angles generates X-rays with different energy. Table 2 shows relation between crossing angle and characteristics of X-rays. Figure 4 shows scattered angle dependence as the function of X-ray energies for certain crossing angles.

Table 2: Maximum energy and number of scattered photons at different crossing angles

\begin{tabular}{|c|c|c|}
\hline $\begin{array}{c}\varphi \\
\text { [degrees] }\end{array}$ & $\begin{array}{c}\max E_{X} \\
{[\mathrm{eV}]}\end{array}$ & $\begin{array}{c}\text { number of scattered photons } \\
\text { [photons/pulse] }\end{array}$ \\
\hline 180 & 450 & $3.8 \times 10^{5}$ \\
160 & 436 & $1.2 \times 10^{5}$ \\
120 & 337 & $3.7 \times 10^{4}$ \\
90 & 225 & $2.1 \times 10^{4}$ \\
\hline
\end{tabular}

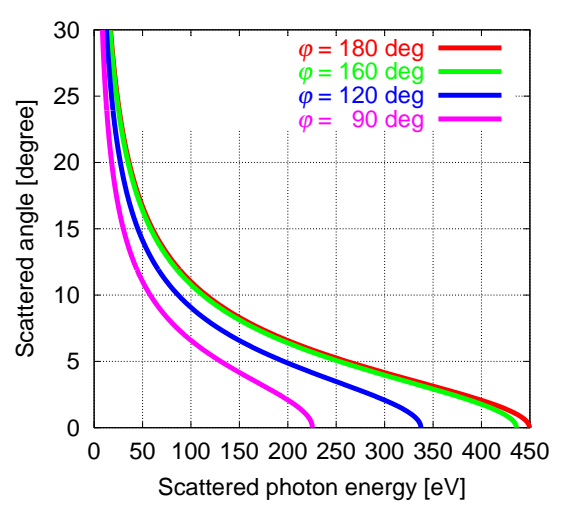

Figure 4: Scattered angle vs scattered photon energy for different crossing angles

So-called "water window" is included in this energy range, then these X-rays are considered to be a powerful tool for biological observation.

\section{SUMMARY}

Timing and intensity stability applicable for the high quality electron beam generation and the inverse Compton scattering are very much improved for our new laser sys- tem. Timing stability is achieved within $0.26 \mathrm{ps}$, and intensity fluctuation is achieved less than $0.11 \%$. We will set the laser system close to rf-gun cavity to obtain better pointing stability on the cathode surface. The intensity stability is affected by the rf field and radiation noises, therefore, we have to add noise shielding box around the laser system for the stable operation. Number of photons, which can be generated by our system, is estimated between $10^{4}-10^{5}$.

In 2001 fiscal year, we will start the operation of rfgun system and will measure the characteristics of electron beam in precise. We will also carry out the X-ray generation experiment using inverse Compton scattering. Before the experiment, we will measure the intensity stability with noise shielding for the laser system.

\section{ACKNOWLEDGMENTS}

Authors would like to express sincere thanks to Professor I. Ben-Zvi and Dr. X. J. Wang of BNL, Drs. A. Endo and K. Takasago of the Femtosecond Technology Research Association for their deep help on the development of the rf-gun and the laser system. This research is supported in part by the Grant for special project of Waseda University, No.99B-029.

\section{REFERENCES}

[1] A. Endo, "Highly stabilized femtosecond Ti:sapphire laser designed for beam interaction experiment", Nucl. Inst. and Meth. A, vol. 455 (2000) No.1 p.228-235

[2] F. Sakai et al., "All-solid-state picosecond laser system for photo cathode rf gun”, PAC'99, New York, March 1999.

[3] H. Tsuchida, "Wideband phase noise measurement of modelocked laser pulse by a demodulation technique", Opt. Lett. 23 (1998) p.286-288

[4] W. Leemans et al., "Femtosecond X-rays from $90^{\circ}$ Thomson scattering”, PAC'95, Dallas, May 1995.

[5] S. Kashiwagi et al., "Observation of high-intensity X-rays in inverse Compton scattering experiment", Nucl. Inst. and Meth. A, vol. 455 (2000) No.1 p.36-40 